\title{
Design of Optimum Gain Pyramidal Horn with Improved Formulas Using Particle Swarm Optimization
}

\author{
Yahya Najjar, Mohammad Moneer, Nihad Dib \\ Electrical Engineering Department, Jordan University of Science and Technology, \\ P.O. Box 3030, Irbid, Jordan \\ Received 5 June 2006; accepted 29 August 2006
}

\begin{abstract}
In this article, a new method to design an optimum gain horn antenna is presented. This method is based on improving existing design equations along with the use of particle swarm optimization technique. The obtained results are more accurate than those available in the literature since no path length error approximation is involved. Improved horn dimensions, for different 15 designs, are obtained which give the desired gain almost exactly. @ 2007 Wiley Periodicals, Inc. Int J RF and Microwave CAE 17: 505-511, 2007.
\end{abstract}

Keywords: horn antenna; particle swarm optimization

\section{INTRODUCTION}

Optimum gain pyramidal horns are widely used as standard gain antennas since their gains can be calculated to within $0.1 \mathrm{~dB}$ using their known dimensions $[1,2]$. An optimum gain pyramidal horn antenna is one that produces maximum gain for given $E$ - and $H$ plane slant lengths. Several methods [1-8] have been presented and used to design a pyramidal horn with optimum gain. Each method has its own accuracy, limitations, and computational complexity. One common feature in [1-8] is the use of the expressions that give optimum gain that are derived from an approximate formula (the first two terms of binomial series expansion) for the path length error that gives rise to aperture quadratic phase error [1]. The method described in [1] is only valid for long, high-gain horns, and uses trial and error (iterative technique). The method proposed in [2] avoids the application of iterative techniques, and is not restricted to high-gain

Correspondence to: N. Dib; e-mail: nihad@just.edu.jo

DOI 10.1002/mmce.20245

Published online 22 June 2007 in Wiley InterScience (www. interscience.wiley.com). horn design. However, the actual gain results do not agree well with the desired gain results. Also, the horn problem was formulated in terms of a fourth degree polynomial that was analytically solved for the narrow aperture dimension. The methods described in [3-5] improved the design equations given in [2] using curve fitting. The method proposed in [6] gives more accurate results than the previous methods, but it needs a large number of inputs and outputs to develop and train a neural network. Recently, in $[7,8]$, the differential evolution technique and the particle swarm optimization method were used to derive new expressions for the narrow and wide aperture dimensions, respectively.

In this article, improved formulas for the relationship between the optimum gain horn aperture size and lengths are derived using the more accurate gain expression given in [9], which does not involve the approximate path length error. These formulas are then used along with the particle swarm optimization technique [10-13] to find directly the physical dimensions of the horn. Our results are compared to those obtained using other techniques [1-8] and shown to give the desired gain exactly.

() 2007 Wiley Periodicals, Inc. 


\section{PYRAMIDAL HORN DESIGN}

Consider the geometry of a pyramidal horn antenna with the $E$-plane $\left(B, B_{1}, L_{\mathrm{E}}, L_{1}, P_{\mathrm{E}}\right)$ and $H$-plane $(A$, $\left.A_{1}, L_{\mathrm{H}}, L_{2}, P_{\mathrm{H}}\right)$ cross-sectional views as shown in Figure 1 . To design a pyramidal horn, one usually knows the desired gain $\mathrm{G}$, the wavelength $\lambda$, and the inner
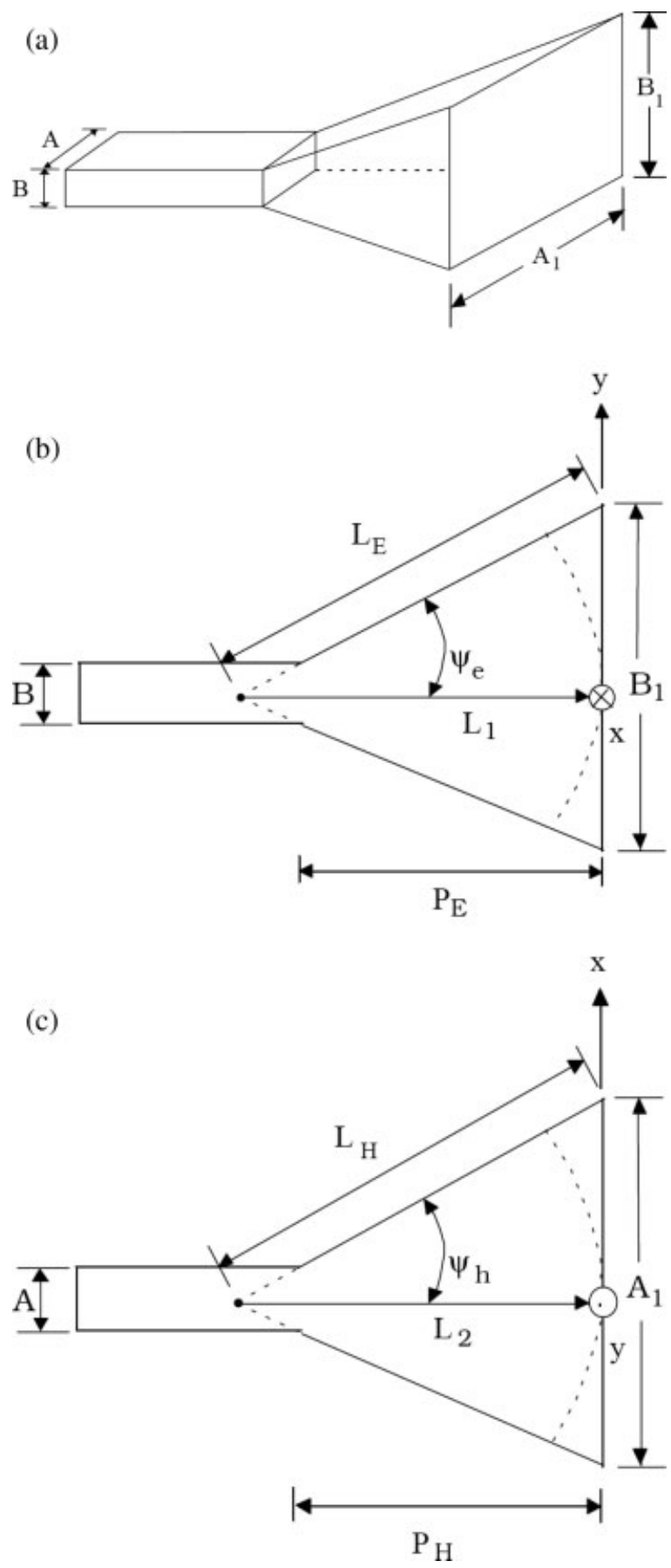

Figure 1. (a) Pyramidal horn antenna (b) E-plane view (c) $H$-plane view. dimensions $A$ and $B$ of the feeding rectangular waveguide. The other parameters $\left(B_{1}, L_{\mathrm{E}}, L_{1}, P_{\mathrm{E}}, A_{1}, L_{\mathrm{H}}\right.$, $L_{2}$, and $P_{\mathrm{H}}$ ) are then determined using a specific design method.

A horn is said to be optimum when the aperture dimensions are adjusted to give maximum gain for given slant lengths in the $E$ - and $H$-planes. According to [1], this occurs when the following relations are satisfied:

$$
\begin{aligned}
& B_{1}=\sqrt{2 \lambda L_{1}} \\
& A_{1}=\sqrt{3 \lambda L_{2}}
\end{aligned}
$$

This means that optimum gain is obtained when the maximum aperture phase deviations in the $E$ - and $H$ planes are $0.25 \lambda$ and $0.375 \lambda$, respectively [1]. The two equations above were obtained by means of curve fitting for $\left(L_{1}, B_{1}\right)$ and $\left(L_{2}, A_{1}\right)$ that give the maximum gain. However, it should be emphasized that the expressions used to derive eqs. (1) and (2) were not exact since the path length error, which gives rise to the quadratic phase error, was approximated by the first two terms of a binomial series expansion of the exact expression [1]. Such an approximation was done to simplify the derivation, which led to closed form expressions for the electric and magnetic fields in terms of cosine and sine Fresnel integrals. In most cases, it yields to very good results for long high gain horns. The above approximate relations were used in all the design methods presented in [1-8]. Here, new improved formulas will be presented as explained below.

In [9], an expression for the gain of pyramidal horn antenna was derived without approximating the path length error. The expression is given by:

$$
\begin{gathered}
G=\frac{32 A_{1} B_{1}}{\pi \lambda^{2}}\left(L_{\mathrm{e}}(S)\right)\left(L_{\mathrm{h}}(T)\right) \\
L_{\mathrm{e}}(S)=\left|\frac{2}{B_{1}} \int_{0}^{B_{1} / 2} e^{-j K L_{1}\left[\sqrt{1+\left(y / L_{1}\right)^{2}}-1\right]} \mathrm{d} y\right|^{2} \\
\left.L_{\mathrm{h}}(T)=\mid \frac{\pi}{A_{1}} \int_{0}^{A_{1} / 2} \cos \left(\frac{\pi x}{A_{1}}\right) e^{-j K L_{2}\left[\sqrt{1+\left(y / L_{2}\right)^{2}}-1\right.}\right]\left.\mathrm{d} x\right|^{2}
\end{gathered}
$$

It is interesting to note that the above expression was used in [2-8] to find the gain of the designed 


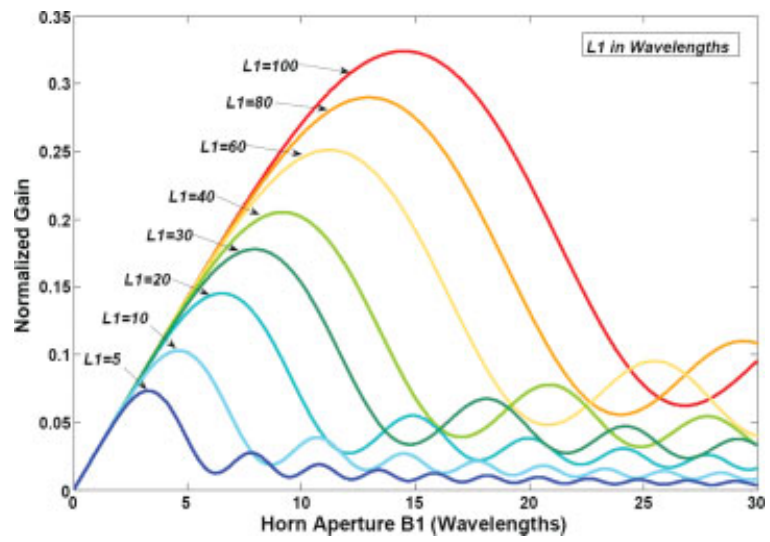

Figure 2. $E$-plane normalized gain of pyramidal horn as a function of $B_{1}$ for different lengths of $L_{1}$. [Color figure can be viewed in the online issue, which is available at www.interscience.wiley.com.]

pyramidal horns, although the design itself involved the use of the approximate eqs. (1) and (2).

Now, using eq. (3), the $E$-plane normalized gain $G_{\mathrm{n}}$ is plotted versus $B_{1}$ for different lengths of $L_{1}$, as shown in Figure 2. The normalized $E$-plane gain $G_{\text {n }}$ is defined as:

$$
G_{\mathrm{n}}=\frac{G \pi \lambda^{2}}{32 A_{1} L_{\mathrm{h}}(T)}
$$

The integrals in eqs. (4) and (5) are evaluated numerically within an error of $10^{-9}$ using an adaptive Gauss/Lobatto quadrature rule. If the values of $B_{1}$ (in $\lambda$ ) which correspond to the maximum gains in Figure 2 are plotted versus their corresponding values of $L_{1}$ (in $\lambda$ ), the following relation can be obtained using curve fitting for 25 points,

$$
B_{1}=\sqrt{2.1060 \lambda L_{1}}
$$

This corresponds to a maximum phase deviation in the $E$-plane of $0.2633 \lambda$.

Figure 3 shows the differences between eqs. (1) and (7) as compared to those obtained directly from eq. (3) using the peaks in Figure 2. It can be seen that eq. (7) gives better results for $B_{1}$ than eq. (1).

Similarly, Figure 4 shows the $H$-plane normalized gain versus $A_{1}$ for different lengths of $L_{2}$ as obtained using eq. (3). Here, the $H$-plane normalized gain $G_{\mathrm{n}}$ is defined as:

$$
G_{\mathrm{n}}=\frac{G \pi \lambda^{2}}{32 B_{1} L_{\mathrm{e}}(S)}
$$

If the values of $A_{1}$ (in $\lambda$ ) which correspond to the maximum gains in Figure 4 are plotted versus their

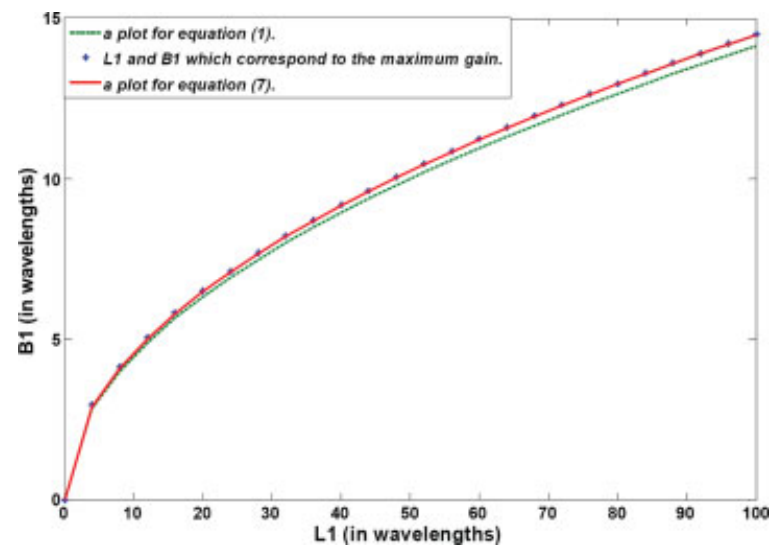

Figure 3. $B_{1}$ versus $\mathrm{L}_{1}$ obtained using eqs. (1) and (7) compared to values obtained from (3). [Color figure can be viewed in the online issue, which is available at www.interscience.wiley.com.]

corresponding values of $L_{2}$ (in $\lambda$ ), the following equation can be obtained using curve fitting for 25 points:

$$
A_{1}=\sqrt{3.1831 \lambda L_{2}}
$$

This corresponds to maximum phase deviation in the $H$-plane of $0.39789 \lambda$. Figure 5 shows the differences between eqs. (2) and (9) as compared to those obtained directly from eq. (3) using the peaks in Figure 4. It can be seen that eq. (9) gives better results for $B_{1}$ than eq. (2).

Recently, in [14], the phases that correspond to the maximum gain in the $E$-plane and $H$-plane of a pyramidal horn antenna were given as $0.26 \lambda$ and $0.4 \lambda$, respectively. Our results obtained using eqs. (7) and (9) agree very well with these values. To our knowledge, the improved formulas (7) and (9) have not appeared in the literature before.

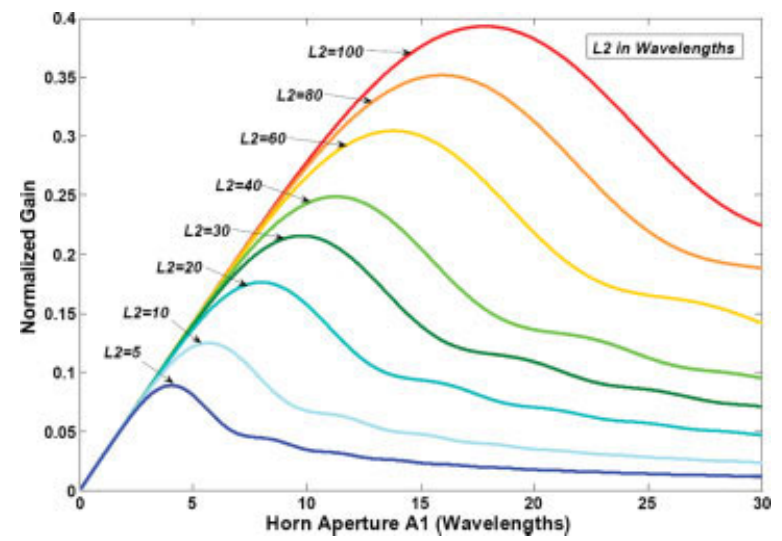

Figure 4. Normalized $H$-plane gain of pyramidal horn as a function of $A_{1}$ for different lengths of $L_{2}$. [Color figure can be viewed in the online issue, which is available at www.interscience.wiley.com.] 


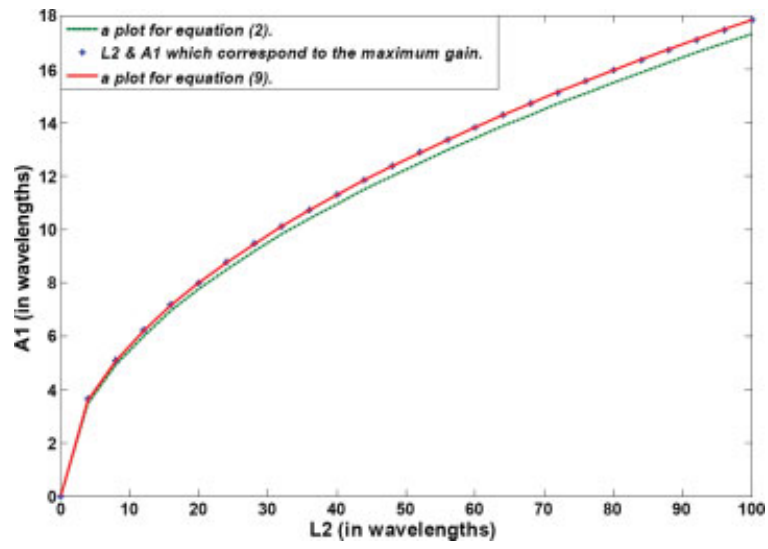

Figure 5. $A_{1}$ versus $L_{2}$ obtained using eqs. (2) and (9) compared to values obtained from (3). [Color figure can be viewed in the online issue, which is available at www.interscience.wiley.com.]

Now, an optimization problem is introduced in which the feeding waveguide dimensions ( $A$ and $B)$, the frequency $(f)$, and the desired optimum gain $(G)$ are given as input parameters, while equation (3) is used to find the dimension $B_{1}$. The parameters $L_{1}, L_{2}$, and $A_{1}$ are given by the following equations:

$$
\begin{gathered}
L_{1}=\frac{B_{1}^{2}}{2.1060 \lambda} \\
L_{2}=\frac{A_{1}^{2}}{3.1831 \lambda} \\
A_{1}=\frac{A+\sqrt{A^{2}-6.0458\left(B B_{1}-B_{1}\right)}}{2}
\end{gathered}
$$

Equation (12) is obtained by enforcing the fact that; for a pyramidal horn to be physically realizable $P_{\mathrm{E}}$ and $P_{\mathrm{H}}$ must be equal. From the geometry of the horn in Figure 1, the following relations for $P_{\mathrm{E}}$ and $P_{\mathrm{H}}$ can be easily derived:

$$
\begin{aligned}
& P_{\mathrm{E}}=\frac{L_{1}\left(B_{1}-B\right)}{B_{1}} \\
& P_{\mathrm{H}}=\frac{L_{2}\left(A_{1}-A\right)}{A_{1}}
\end{aligned}
$$

So, our optimization problem here is a one-dimensional problem. The particle swarm optimization (PSO) method is chosen to solve the problem since; recently, we have been interested in the application of PSO in different electromagentics and microwave circuits problems $[12,13]$. The PSO technique has been successfully applied to antenna design $[8,10$,
11], and the results proved that this method is powerful and effective for optimization problems. PSO is similar in some ways to genetic algorithms, but requires less computational bookkeeping and generally fewer lines of code, including the fact that the basic algorithm is very easy to understand and implement. The interested reader can refer to $[8$, 10-13], and the references therein, for details of the PSO algorithm. It should be noted that our application of the PSO differs completely from that presented in [8]. In [8], a closed form expression, which involves 11 constants, for the wider dimension $A_{1}$ was derived using the PSO; while in our approach the gain formula (3) is directly optimized using the PSO.

In our implementation of the PSO, $B_{1}$ that gives the optimum gain is found using the following fitness function:

$$
\text { Fitness }=\text { Equation }(3)-G_{\text {desired }}
$$

\section{RESULTS}

Table I shows 15 different horn design examples, which have been used to compare our PSO results to those obtained using the previous design methods [1-8].

First, to check the validity of our design approach and to compare with the results presented in [6] (since they are the most accurate among [18]), eq. (3) has been solved using PSO while using the approximate formulas (1) and (2) instead of the improved formulas (7) and (9). This is done so that we have a fair comparison since (1) and (2) were used in [6]. Specifically, the following equations for $L_{1}, L_{2}$, and $A_{1}$ are used instead of equations (10)-(12):

$$
\begin{gathered}
L_{1}=\frac{B_{1}^{2}}{2 \lambda} \\
L_{2}=\frac{A_{1}^{2}}{3 \lambda} \\
A_{1}=\frac{A+\sqrt{A^{2}-6\left(B B_{1}-B_{1}\right)}}{2}
\end{gathered}
$$

Our results for the wider dimension $B_{1}$ along with those reported in [6] are shown in Table II. In [6], a neural network was trained to find the value of the dimension $B_{1}$, while it is found directly here using PSO. The desired values for $B_{1}$ are also taken 
TABLE I. Values of Frequencies and Inner Dimensions of Common Standard Rectangular Feed Waveguides and Desired Gains

\begin{tabular}{|c|c|c|c|c|c|c|}
\hline \multirow{2}{*}{$\begin{array}{c}\text { Example } \\
\text { No. }\end{array}$} & \multicolumn{2}{|c|}{$\begin{array}{l}\text { Waveguide } \\
\text { Standard }\end{array}$} & \multirow{2}{*}{$\begin{array}{l}\text { Frequency } \\
\qquad(\mathrm{GHz}) f\end{array}$} & \multirow{2}{*}{$\begin{array}{c}\text { Desired } \\
\text { Gain }(\mathrm{dB}) G\end{array}$} & \multicolumn{2}{|c|}{$\begin{array}{c}\text { Waveguide } \\
\text { Dimensions }(\mathrm{cm})\end{array}$} \\
\hline & I EC & WR & & & $A$ & $B$ \\
\hline 1 & R14 & WR650 & 1.431 & 15.85 & 16.51 & 8.255 \\
\hline 2 & $\mathrm{R} 22$ & WR430 & 2.163 & 16.50 & 10.95 & 5.461 \\
\hline 3 & $\mathrm{R} 26$ & WR340 & 2.736 & 17.40 & 8.636 & 4.318 \\
\hline 4 & R32 & WR284 & 3.275 & 18.03 & 7.214 & 3.404 \\
\hline 5 & $\mathrm{R} 40$ & WR229 & 4.061 & 18.80 & 5.817 & 2.908 \\
\hline 6 & $\mathrm{R} 48$ & WR187 & 4.968 & 19.95 & 4.755 & 2.215 \\
\hline 7 & R58 & WR159 & 5.849 & 20.85 & 4.039 & 2.014 \\
\hline 8 & R70 & WR137 & 6.779 & 21.75 & 3.485 & 1.580 \\
\hline 9 & $\mathrm{R} 84$ & WR112 & 8.290 & 22.50 & 2.850 & 1.262 \\
\hline 10 & R100 & WR90 & 10.34 & 22.70 & 2.286 & 1.016 \\
\hline 11 & R120 & WR75 & 12.40 & 23.20 & 1.905 & 0.953 \\
\hline 12 & $\mathrm{R} 140$ & WR62 & 14.95 & 23.50 & 1.580 & 0.790 \\
\hline 13 & R180 & WR51 & 18.24 & 23.85 & 1.295 & 0.648 \\
\hline 14 & $\mathrm{R} 280$ & WR34 & 27.36 & 24.30 & 0.864 & 0.432 \\
\hline 15 & R320 & WR28 & 33.22 & 24.60 & 0.711 & 0.356 \\
\hline
\end{tabular}

from [6]. According to [15], these desired values were first obtained roughly using previously published formulas [3-5], then the desired $B_{1}$ values, which lead to an excellent agreement between the computed gains of the designed pyramidal horns and the desired gains, were obtained by using an iterative technique. It can be seen that the PSO results are in excellent agreement with the desired $B_{1}$ values.

Having verified the design approach, the improved formulas (7) and (9) are now used to find the wider dimension $B_{1}$ using PSO. Our improved results for $A_{1}$ and $B_{1}$ are given in Table III along with our results from Table II. It can be seen that there are small differences between the two sets of results. The difference ranges from $2 \mathrm{~mm}$ for the highest gain example to around $1.5 \mathrm{~cm}$ for the lowest gain example. We believe that these small differences are very important since exact dimensions are usually needed for horn antennas when used as a standard gain antenna to determine the precise gain of other antennas.

TABLE II. A Comparison of the Desired $B_{1}$ Values with the Results Reported in [6] and Those Obtained Using the PSO with eqs. (1) and (2)

\begin{tabular}{|c|c|c|c|c|c|}
\hline \multirow[b]{2}{*}{ No. } & \multirow[b]{2}{*}{ Desired $B_{1}(\mathrm{~m})$} & \multicolumn{2}{|c|}{ Guney [6] } & \multicolumn{2}{|c|}{ Our Results Using PSO } \\
\hline & & $B_{1}(\mathrm{~m})$ & Absolute Error & $B_{1}(\mathrm{~m})$ & Absolute Error \\
\hline 1 & 6108 & . & 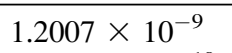 & & . \\
\hline 2 & 144154246448677 & 1441542405646650 & $5.8840 \times 10^{-10}$ & 0.31441542464479 & $7.771561172376096 \times 10^{-14}$ \\
\hline 3 & 2774569102970922 & 27745690986449739 & $4.3259 \times 10^{-10}$ & 0.27745691029704 & $5.218048215738236 \times 10^{-14}$ \\
\hline 4 & 09338 & 496690151894 & $8.7397 \times 10^{-7}$ & 0.24966814122088 & $5.107025913275720 \times 10^{-}$ \\
\hline 5 & 0.22140 & 22140310964312 & $7.2819 \times 10^{-11}$ & 0.22140310957023 & $6.772360450213455 \times 10^{-1}$ \\
\hline 6 & 028 & 072801178764 & $3.8752 \times 10^{-10}$ & 0.20728011748883 & $7.444045380111675 \times 10^{-1}$ \\
\hline 7 & 91 & 6 & $6.6018 \times 10^{-10}$ & 174725 & $7.463474283042615 \times 10^{-1}$ \\
\hline 8 & 76 & 7 & $7.8573 \times 10^{-10}$ & 1853804 & $1.679489880501706 \times 10^{-1}$ \\
\hline 9 & 386 & 711 & $4.3199 \times 1$ & 9572227 & $7.233103005432895 \times 10^{-1}$ \\
\hline 10 & 2667574 & & $1.1736 \times 10^{-9}$ & 0.13789581426670 & $5.698219673888616 \times 10^{-1}$ \\
\hline 11 & 22429966 & 1222429 & $1.3465 \times 10^{-9}$ & 0.122242 & $5.150047055479945 \times 10^{-}$ \\
\hline 12 & & & $9.2607 \times 10$ & 0.105029 & $4.475586568020162 \times 10^{-}$ \\
\hline 13 & 0.0896947535384475 & 0.08969475492360850 & $1.3855 \times 10^{-9}$ & 0.08969475353841 & $3.851086116668512 \times 10^{-}$ \\
\hline 14 & & 0.06303561728956132 & $1.4027 \times 10^{-9}$ & 0.06303561588684 & $2.735311976920229 \times 10^{-}$ \\
\hline 15 & 0.0537725214751637 & 0.05377252230591544 & $8.3075 \times 10^{-10}$ & 0.05377252147514 & $2.341876692568690 \times 10^{-1}$ \\
\hline
\end{tabular}


TABLE III. The Aperture Dimensions (in meters) for the 15 Experiments Given in Table I

\begin{tabular}{|c|c|c|c|c|}
\hline \multirow[b]{2}{*}{ No } & \multicolumn{2}{|c|}{ PSO with Improved Formulas } & \multicolumn{2}{|c|}{ PSO with Formulas (1) and (2) } \\
\hline & $\begin{array}{c}\text { Aperture } \\
\text { Dimension } A_{1}\end{array}$ & $\begin{array}{c}\text { Aperture } \\
\text { Dimension } B_{1}\end{array}$ & $\begin{array}{c}\text { Aperture } \\
\text { Dimension } A_{1}\end{array}$ & $\begin{array}{c}\text { Aperture } \\
\text { Dimension } B_{1}\end{array}$ \\
\hline 1 & 0.58745939957790 & 0.44853806855461 & 0.57361597193408 & 0.43867213531014 \\
\hline 2 & 0.41897752185742 & 0.32160260813165 & 0.40904904300191 & 0.31441542464479 \\
\hline 3 & 0.36748291047856 & 0.28391860822711 & 0.35840552867341 & 0.27745691029704 \\
\hline 4 & 0.33732363443676 & 0.26089097456624 & 0.32252114892473 & 0.24966814122088 \\
\hline 5 & 0.29088895881357 & 0.22667311271466 & 0.28348102783180 & 0.22140310957023 \\
\hline 6 & 0.27191636671072 & 0.21228861617687 & 0.26486821720179 & 0.20728011748883 \\
\hline 7 & 0.25560936927822 & 0.20111475647621 & 0.24889199855866 & 0.19634325174725 \\
\hline 8 & 0.24504175271475 & 0.19266814169802 & 0.23855032644736 & 0.18805991853804 \\
\hline 9 & 0.20747207152755 & 0.16317793605405 & 0.21260597834625 & 0.16797169572227 \\
\hline 10 & 0.17916414178191 & 0.14129224072333 & 0.17437783238506 & 0.13789581426670 \\
\hline 11 & 0.15784776775620 & 0.12525307711374 & 0.15360236582933 & 0.12224299668255 \\
\hline 12 & 0.13550390047196 & 0.10761884737834 & 0.13185422799275 & 0.10502965303752 \\
\hline 13 & 0.11560862074174 & 0.09190862517665 & 0.11248356493265 & 0.08969475353841 \\
\hline 14 & 0.08115133942015 & 0.06459365453271 & 0.07895527928804 & 0.06303561588684 \\
\hline 15 & 0.06917393039352 & 0.05510271231507 & 0.06729446076815 & 0.05377252147514 \\
\hline
\end{tabular}

Using the dimensions in Table III, the gain is computed using eq. (3) and the improved formulas (7) and (9). The results are shown in Table IV. It can be seen that there is a difference between the desired and the computed gains when using the aperture dimensions obtained using eqs. (1) and (2). Using the improved aperture dimensions $A_{1}$ and $B_{1}$, the desired gain is obtained exactly.

TABLE IV. Computed Gains Using (3) Along with (7) and (9)

\begin{tabular}{cccc}
\hline & $\begin{array}{c}\text { Gain Computed } \\
\text { Using the Aperture } \\
\text { Dimensions } \\
\text { Obtained Using } \\
\text { No. }\end{array}$ & $\begin{array}{c}\text { Gain Computed } \\
\text { Using the }\end{array}$ & \\
\hline 1 & 15.65225377151011 & 15.85000000000000 & 15.85 \\
2 & 16.30503868563279 & 16.50000000000000 & 16.50 \\
3 & 17.19774296438053 & 17.40000000000000 & 17.40 \\
4 & 17.82432108495824 & 18.03000000000000 & 18.03 \\
5 & 18.59070601292597 & 18.80000000000000 & 18.80 \\
6 & 19.73616544011127 & 19.95000000000000 & 19.95 \\
7 & 20.63332003114509 & 20.85000000000000 & 20.85 \\
8 & 21.53096300646700 & 21.75000000000000 & 21.75 \\
9 & 22.27939335994748 & 22.50000000000000 & 22.50 \\
10 & 22.47893636158694 & 22.70000000000000 & 22.70 \\
11 & 22.97793385306501 & 23.20000000000000 & 23.20 \\
12 & 23.27751657819921 & 23.50000000000000 & 23.50 \\
13 & 23.62681934326002 & 23.85000000000000 & 23.85 \\
14 & 24.07637655171835 & 24.30000000000000 & 24.30 \\
15 & 24.37570568796014 & 24.60000000000000 & 24.60 \\
\hline
\end{tabular}

\section{CONCLUSIONS}

New improved formulas for the design of optimum gain horn antennas have been proposed. These formulas do not approximate the path length error, and thus, they give more accurate horn dimensions for a given gain. The particle swarm optimization technique has been used to find the wider dimension directly from the exact gain formula. Better accuracy with respect to previous design methods has been obtained for various design examples.

\section{REFERENCES}

1. C.A. Balanis, Antenna theory: Analysis and design, Wiley, New York, 1997.

2. K.T. Selvan, Accurate design method for optimum gain pyramidal horns, Electron Lett 35 (1999), 249-250.

3. K. Guney, Improved design method for optimum gain pyramidal horns, Int J RF Microwave Comput-Aided Eng 11 (2001), 188-193.

4. K. Guney, A new design method for optimum gain pyramidal horns, Electromagnetics 21 (2001), 497505.

5. K. Guney and H. Hancer, Improved formulas for narrow and wide aperture dimensions of optimum gain pyramidal horn, Int J RF Microwave Comput-Aided Eng 13 (2003), 239-245.

6. K. Guney and N. Sarikaya, Artificial neural networks for the narrow aperture dimension calculation of optimum gain pyramidal horns, Electrical Eng 86 (2004), $157-163$. 
7. K. Guney and D. Karaboga, New narrow aperture dimension expressions obtained using differential evolution algorithm for optimum gain pyramidal horns, J Electromagn Waves Appl 18 (2004), 321-339.

8. A. Akdagli and K. Guney, New wide-aperture-dimension formula obtained by using a particle swarm optimization for optimum gain pyramidal horns, Microwave Opt Technol Lett 48 (2006), 1201-1205.

9. M.J. Maybell and P.S. Simon, Pyramidal horn gain calculation with improved accuracy, IEEE Trans Antennas Propag 41 (1993), 884-889.

10. J. Robinson and Y. Rahmat-Samii, Particle swarm optimization in electromagnetics, IEEE Trans Antennas Propag 52 (2004), 397-407.
11. M.M. Khodier and C.G. Christodoulou, Linear array geometry synthesis with minimum sidelobe level and null control using particle swarm optimization, IEEE Trans Antennas Propag 53 (2005), 2674-2679.

12. J. Ababneh, M. Khodair, and N. Dib, Synthesis of interdigital capacitors based on particle swarm optimization and artificial neural networks, Int J RF Microwave Comput-Aided Eng 16 (2006), 322-330.

13. Nihad Dib and Jehad Ababneh, Physical modeling and particle swarm design of coplanar waveguide square spiral inductor, Int J Model Simul (in press).

14. T.A. Milligan, Modern antenna design, 2nd ed., Wiley, 2005, p. 343.

15. K. Guney, personal communication.

\section{BIOGRAPHIES}

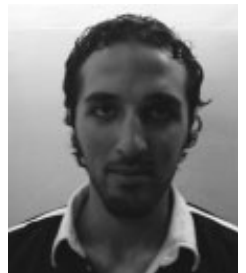

Yahya Najjar was born in Kuwait in March 1984. He is currently studying for B.Sc. in electrical engineering at Jordan University of Science and Technology, Jordan. His fields of interest include mobile communications systems, PSO, antennas, and microwave systems.

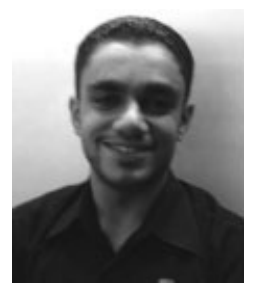

Mohammad Moneer was born in Kuwait in December 1983. He is currently studying for B.Sc. in electrical engineering at Jordan University of Science and Technology, Jordan. His fields of interest include EM and microwave communications, antennas, GSM systems, and computer networks.

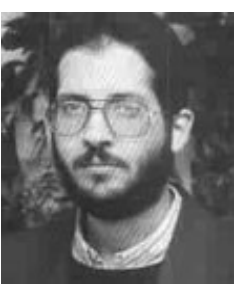

Nihad Dib obtained his B.Sc. and M.Sc. in EE from Kuwait University in 1985 and 1987, respectively. He obtained his Ph.D. in EE (major in Electromagnetics and Microwaves) in 1992 from University of Michigan, Ann Arbor. Then, he worked as an assistant research scientist in the radiation laboratory at the same school. In September 1995, he joined the EE Department at Jordan University of Science and Technology (JUST) as an assistant professor, and became a full professor in August 2006. His research interests are in computational electromagnetics and modeling of planar circuits. 